\title{
Mención cualificadora en inteligencia emocional del Grado de Magisterio en Educación Infantil
}

\author{
Emotional Inteligencia in Inicial Teacher Training (Inicial Tea- \\ cher Training
}

CLAUDIA RUIZ

Departamento de Psicología. Centro de Magisterio "Virgen de Europa"

LUISA Ma GARCÍA

Departamento de Psicología. Centro de Magisterio "Virgen de Europa"

\section{RESUMEN}

El marco social en el que nos desenvolvemos en la actualidad refleja continuos cambios en distintas áreas de desarrollo humano (laboral, económico, político, tecnológico...), exige constantes esfuerzos de adaptación personal y conlleva diferencias significativas respecto a épocas anteriores en los modos de relacionarnos con los demás y con nosotros mismos. Desde diversas tendencias teóricas, se confirma la relevancia de las competencias socioemocionales para enfrentarnos a estas situaciones vitales inestables de modo eficaz. Y la educación se ha hecho eco de esta necesidad. En un contexto de interés nacional e internacional creciente por la Inteligencia Emocional, el Centro de Magisterio Virgen de Europa (adscrito a la Universidad de Cádiz) apostó por una formación inicial que ofreciera en una Mención Cualificadora del Grado de Magisterio en Educación Infantil, la posibilidad de analizar y fomentar el desarrollo socioemocional de los niños y del propio docente. Organizamos la mención en torno a 3 asignaturas (teórico-prácticas) que inciden de forma complementaria en el campo de la inteligencia emocional de los futuros maestros.

\section{ABSTRACT}

The social framework in which we operate today reflects continuous changes in different areas of human development (labor, economic, political, technological...), requires constant efforts of personal adaptation and involves significant differences from previous periods in the ways of relating to others and ourselves. From different theoretical tendencies, confirms the relevance of socio-emotional skills in order to face these unstable life situations effectively. And education has echoed this need. In a context of increasing national and international interest in emotional intelligence, we opted (in our Teacher Training Center Virgen de Europa - attached to the University of Cádiz) for an initial training that offers the possibility of analyzing and promoting the socio-emotional development of children and the teacher himself. We organize teaching around three subjects (theoretical and practical) that affect in a complementary way the field of emotional intelligence of future teachers.

Para citar este artículo: Ruiz, C. y García, L. M. (2019). Mención cualificadora en inteligencia emocional del Grado de Educación Infantil. EA, Escuela Abierta, 22, 3-13. doi:10.29257/ EA22.2019.02

Recibido: 19/09/18

Aceptado: 29/07/19

PALABRAS CLAVES Psicología de la Educación, formación inicial docente, inteligencia emocional, competencias socioemocionales

\section{KEYWORDS}

Educational Psychology, Initial Teacher Training, Emotional Intelligence, Socio-Emotional Skills 


\section{INTRODUCCIÓN}

La redacción de este artículo responde a la necesidad de compartir la experiencia que se nos ha brindado de trabajar en el campo de la inteligencia emocional dentro del contexto universitario formativo de los futuros docentes de Educación Infantil, en particular, e indirectamente de Educación Primaria.

El campo de la inteligencia emocional responde a los planteamientos más actuales que conciben la educación como un proceso influenciado por aspectos que van mucho más allá de lo estrictamente cognitivo o académico y que se traduce en el requerimiento de competencias en este área. Se pretende, además, que los procesos educativos reviertan en la promoción de mejoras sociales y personales (en el mundo actual, globalizado y cambiante, se considera necesario fomentar el bienestar personal y social de las personas para posibilitar la prevención de la violencia y de las conductas de riesgo, la gestión de los conflictos interpersonales y la promoción de la resiliencia, tan necesaria para afrontar las dificultades y los cambios continuos).

Así, los futuros docentes necesitan tener una formación teórica mínima y unas competencias propias a nivel socioemocional que les capaciten para enfrentarse a lo que la educación del alumnado del siglo XXI reclama: una formación integral que contemple la faceta emocional del individuo. López Cassá (2012) se hace eco de este planteamiento. Las emociones, además de ser relevantes per sé para el desarrollo personal, están íntimamente relacionadas con otros aspectos abarcados tradicionalmente en la enseñanza (evidentemente, nos referimos a los procesos cognitivos y de aprendizaje en las diferentes disciplinas académicas). Y así se recoge en los informes de la UNESCO que, entre otras temáticas, abordan el tema de la incorporación explícita de contenidos socioemocionales y la necesidad de la modificación del rol docente en este sentido.

\section{LA ESCUELA DE LA INTELIGENCIA EMOCIONAL}

Si nos remontamos en el tiempo, la Teoría de las Inteligencias Múltiples de Gardner (difundida en 1983) (Gardner, 1999) ya permite intuir el peso y la relevancia que en el campo de la psicología se va dando a la inteligencia emocional (cuando recoge entre todas las inteligencias que identifica en el ser humano- lógico-matemática, lingüistica, espacial, musical, corporal-kinestésica, naturalista, interpersonal, intrapersonal y existencial- las tres últimas, según nuestro punto de vista, se pueden relacionar claramente con la inteligencia emocional). Estas inteligencias estarían presentes en todas las personas aunque en un grado diferente en función de factores biológicos (de carácter genético), psicológico (disposiciones personales relacionadas con la motivación, la creatividad..) o sociales (provenientes de los valores culturales, familiares o escolares e implican la transmisión social y familiar).

Mayer y Salovey (1990) describen el concepto de inteligencia emocional y Goleman (1998) lo da a conocer a la población general en su famoso best seller del mismo nombre. Y desde ese momento, es un campo que se estudia en profundidad y que culmina en la toma de conciencia de que es necesario poseer un conjunto de competencias emocionales, definidas por Navarro y Martín (2010) como "los conocimientos, habilidades, actitudes, procedimientos y comportamientos que permiten comprender, expresar y regular de forma adecuada los fenómenos emocionales" (p. 62). Puntualizamos que este conjunto de conocimientos es necesario conocerlos en un doble sentido: personal (y las potenciales aplicaciones sobre uno mismo) y social (sobre los demás).

Por tanto, introducir esta temática en la formación del alumnado del Centro de Magisterio Virgen de Europa es innovador por lo que supone respecto a las aportaciones científicas actuales (neurociencias, psicología, pedagogía) y se traduciría en buenas prácticas educativas en el futuro maestro; por ello, consideramos importante integrar estos contenidos en la formación inicial de las generaciones de maestros que ejercerán en años próximos. 
Se podría concluir que la inteligencia emocional es la capacidad para reconocer y manejar nuestros propios sentimientos, la capacidad de motivarnos y dirigir nuestro comportamiento en pro de unas relaciones sanas y satisfactorias. Aspectos relacionados con la cognición (entendida en el sentido más clásico de comprensión de los fundamentos de un hecho ya sea de carácter académico o social), la motivación personal, la personalidad de cada uno y los aspectos biológicos influirían en este tipo de inteligencia y serían susceptibles de ser educados (Maganto y Maganto, 2010). La utilidad del conocimiento de las emociones se relacionaría con factores como:

- Nos ayudan a adaptarnos a las circunstancias y a protegernos ante situaciones potencialmente peligrosas (o a acercarnos a situaciones beneficiosas para la adaptación y la supervivencia).

- Nos proporcionan información sobre nuestro estado y reacciones ante sucesos significativos, sobre el estado de los demás y les permite a los otros obtener información sobre nosotros mismos.

- Es por ello que cumplen una función social: facilitan la relación social y la comunicación entre los integrantes de una comunidad (ya sea familiar, escolar, amistosa...).

- Además cumplen una función motivacional: preparan para dirigir la conducta hacia el acercamiento a aquello que nos parece atractivo o necesario y el alejamiento de aquello que nos parece potencialmente peligroso.

Se puede apreciar que son vitales las emociones para situarse en los marcos sociales de referencia, sean del tipo que sean. Pero, indudablemente, que sean eficaces y permitan una adaptación personal y social, depende del entrenamiento o estimulación que de las mismas se haga y del momento de desarrollo en el que nos encontremos. En los casos en que las emociones no se gestionan adecuadamente se dan situaciones desadaptativas como: respuestas que alteran el bien social (con el consiguiente rechazo de los demás), incapacidad de resolución de conflictos, escasa capacidad de tolerancia a la frustración o de adaptación, pueden producirse alteraciones de las funciones corporales (somatización de problemas que derivan en trastornos físicos) o de funciones mentales, sociales o laborales. Dejando de lado aquellos casos en los que se trate de patologías identificables (que serían objeto de intervención especializada), se abre un amplio abanico de posibilidades a la educación de las emociones que ayuden a la gestión de la cotidianidad.

El proceso de formación en este campo pasaría por una serie de fases que iría desde la propia toma de conciencia del estado emocional de cada uno, el autocontrol del mismo, la toma de conciencia social hasta el manejo de las relaciones con los demás. Se traducirían estas habilidades en una mejor preparación para la vida. Por lo que sería coherente que la escuela contemplara esta formación, con el docente como guía de la misma. Concretamente, los objetivos de la escuela en este campo se relacionarían con:

- La detección de posibles casos de pobre desempeño emocional, por parte del docente, en etapas tempranas del desarrollo.

- El fomento del conocimiento de las emociones en uno mismo y en los demás.

- La enseñanza del modo adecuado de expresión de los estados de ánimo.

- El desarrollo de la empatía.

- El desarrollo de la tolerancia a la frustración.

- La prevención de las conductas de riesgo. 
- La adopción de una actitud positiva ante la vida.

- La gestión de los conflictos interpersonales...

Como se puede apreciar, estos planteamientos se relacionarían también con otros como la escuela inclusiva, requisito indispensable en una sociedad plural que respeta y fomenta el respeto hacia la diferencia.

El interrogante que se plantea es el siguiente: ¿cómo pueden los docentes cuya formación emocional ha quedado en manos del azar planificar una enseñanza y una actuación en este campo? Los datos demuestran que en la enseñanza los niveles de estrés son altos, desembocando en el llamado burn out (síndrome del quemado) y en un número de bajas anuales considerables relacionadas con el estrés y la ansiedad. Se puede considerar, pues, que son muchos los docentes que no poseen las herramientas de gestión de las emociones necesarias. Si analizamos cuáles son los estresores de esta profesión, apreciamos que se relacionan con aspectos como:

- La falta de motivación del alumnado.

- Los problemas de disciplina en el aula.

- La falta de tiempo en el horario escolar para la preparación de las clases (sobre todo en determinados momentos del curso).

- La sobrecarga, determinada también por los requerimientos administrativos.

- Los frecuentes cambios en la legislación que obligan a un reciclaje constante.

- Las modificaciones en el rol docente (casi siempre con más funciones de las que se reconocen).

- La falta de apoyo entre los miembros del equipo docente o directivo ante situaciones concretas, incluso de los padres.

- El escaso reconocimiento social de la profesión (más si lo comparamos con épocas anteriores)...

Se hace evidente la necesidad de que el propio profesorado reciba formación en este campo: que aprenda el modo de adoptar una actitud alternativa (lo que Bermejo, 2016, denomina engagement- compromiso, vigor, absorción) en los momentos adecuados. Y que adquiera una serie de competencias emocionales vinculadas a su profesión con conocimientos básicos desde un punto de vista teórico y estrategias de auto-observación para el estímulo de la propia conciencia (Bayón, 2015).

Vaello (2011) recoge los resultados que obtendría el docente emocionalmente inteligente:

- Desarrolla una autoestima sana.

- Es más competente profesionalmente, traduciéndose en una enseñanza de mayor calidad.

- Tiene menos problemas de disciplina en sus aulas.

- Es más empático.

- Resiste mejor las presiones laborales.

- Supera las adversidades con mayor eficacia (relacionado con el concepto de resiliencia). 
Por ello tendrá más probabilidades de sentirse feliz y realizado en su contexto personal y laboral, con lo cual se incrementaría su eficacia real y percibida.

De esta justificación parte nuestro trabajo como docentes universitarias que imparten asignaturas en una mención cualificadora en el Centro de Magisterio Virgen de Europa llamada "Comportamiento prosocial y habilidades socioemocionales en Educación Infantil” en la que incidiremos más adelante.

\section{DEMANDAS FORMATIVAS PARA SER UN DOCENTE EMOCIONALMENTE INTELI- GENTE}

Desde el momento en que se implantaron los estudios de Grado y se revisaron los planes docentes formativos de los estudios de magisterio, se planteó en nuestro centro la posibilidad de trabajar en la temática descrita anteriormente. Así, en los estudios de Grado de Magisterio en Educación Infantil se ofreció la posibilidad de diseñar una mención que (a través de la elección de las optativas correspondientes) posibilitara al alumnado la especialización en este campo. El hecho de que se trate de optativas tiene como principal ventaja que los alumnos que las cursan, lo hacen porque están interesados en la temática (ofertada por el Departamento de Psicología del nuestro centro). El inconveniente: que no todos los alumnos cursarán la mención por lo que, aunque este tipo de contenidos se revelan como imprescindibles en la enseñanza, no todos los futuros docentes estarán formados al mismo nivel.

Nuestro alumnado proviene de la provincia de Cádiz (fundamentalmente de la comarca del Campo de Gibraltar) y localidades cercanas de la provincia de Málaga. En general, el Grado en Educación Infantil es elegido por mujeres, mayoritariamente procedentes de bachillerato de sociales o humanidades (algunas acceden desde el Ciclo Formativo Superior de Educación Infantil). La elección del Grado en Educación Infantil suele estar basada en la vocación por la docencia y en la enseñanza a los más pequeños.

Por otro lado, la mención es seleccionada por alumnas con inquietudes en el área de la psicología y con deseos de innovar en la docencia en esta etapa educativa, partiendo del perfil evolutivo del niño en todas sus facetas y asumiendo la interrelación entre lo socioemocional y lo cognitivo. En los últimos años hemos asistido a un descenso en el número de alumnos de este grado (en parte ocasionado por la falta de plazas de oposiciones para esta etapa educativa), y sin embargo, hay un aumento de interés en el tema, que se refleja en el número de matriculados en las optativas de la mención en comparación con el total de alumnos de nuestro centro.

La denominación de la mención es la siguiente: Comportamiento prosocial y habilidades socioemocionales en Educación Infantil. Se compone de tres asignaturas (todas de 6 créditos, 2 prácticos y 4 teóricos) que en conjunto, dan una visión completa de la temática, aunque somos conscientes de que no agotan ni garatizan la formación en este campo (puede profundizarse en la mención y asignaturas en la página web de nuestro centro www.magisteriolalinea. com ). Además, la asignatura Prácticum II de cuarto curso, posee un área de aplicación práctica de la mención. Profundicemos en cada una de las tres asignaturas descritas: Habilidades socioemocionales y relaciones entre iguales en Educación Infantil (asignatura 1): esta asignatura se imparte en el segundo semestre del tercer curso de los estudios de magisterio en Ed. Infantil.

- Los contenidos están relacionados con las nociones básicas, de corte más teórico, sobre emociones y neurociencia, inteligencia emocional, el desarrollo social y emocional del niño, las correlaciones entre lo biológico y lo cognitivo-conductual (neurociencia), las influencias en el desarrollo socioemocional y la evaluación del 
comportamiento socioemocional en el aula. Se promueve el debate y la participación activa del alumnado, con el fin de que construya un conocimiento propio sobre el área y lo relacione con sus propias vivencias (en el momento de impartición se encuentran realizando la asignatura Prácticum I en los centros colaboradores, que aunque es de carácter generalista, proporciona un contexto práctico sobre el que aplicar la teoría).

- En la Tabla 1 que presentamos a continuación, se recogen las competencias que se pretenden desarrollar en el alumnado de magisterio con esta asignatura.

\section{Tabla 1}

Competencias de la asignatura Habilidades socioemocionales y relaciones entre iguales en Educación Infantil

\begin{tabular}{|c|c|}
\hline \multirow{5}{*}{$\begin{array}{l}\text { Habilidades } \\
\text { socioemociona- } \\
\text { les y relaciones } \\
\text { entre iguales en } \\
\text { Ed. Infantil }\end{array}$} & $\begin{array}{l}\text { la calidad de las relaciones que se establecen con los iguales } \\
\text { la }\end{array}$ \\
\hline & $\begin{array}{l}\text { CEO19 Reflexionar sobre el papel de unas adecuadas relaciones con los iguales en el ajuste } \\
\text { socioemocional y la adaptación escolar }\end{array}$ \\
\hline & $\begin{array}{l}\text { CG2 Conocer los desarrollos de la psicología evolutiva de la infancia en los periodos 0-3 y } \\
\text { 3-6. }\end{array}$ \\
\hline & $\begin{array}{l}\text { CG15 Conocer los principios básicos de un desarrollo y comportamiento saludables Gene- } \\
\text { ral }\end{array}$ \\
\hline & $\begin{array}{l}\text { limenticias y de bienestar que perturben el desarrol } \\
\text { udiantes }\end{array}$ \\
\hline
\end{tabular}

- Actividades para fomentar el desarrollo socioemocional en Educación Infantil (asignatura 2): al igual que la anterior, esta asignatura se imparte en el segundo semestre del curso $3^{\circ}$ y se simultanea también, por tanto, con el periodo de Prácticum I.

El hecho de que tanto esta asignatura como la anterior, se impartan en el mismo periodo implica un importante esfuerzo de coordinación entre las profesoras para dar sentido a los contenidos que se trabajan en una y otra y que se complementen.

En este caso, el enfoque de la asignatura es eminentemente práctico. Pretendemos que el alumnado analice los programas de desarrollo socioemocional y recursos existentes para la etapa de Educación Infantil y elaboren algunas propuestas personales en base a los estudios de diversos autores (el diseño de actividades y recursos para el desarrollo de las competencias socioemocionales: conciencia emocional, autonomía emocional, regulación emocional, etc). Se promueve el trabajo cooperativo entre el alumnado, la puesta en común de las actividades, y el análisis de las mismas. Resulta especialmente interesante que estén cursando en este momento su primer periodo de prácticas pues permite al alumnado aplicar en la realidad (de un modo más o menos voluntario) lo que están viendo en el Centro Universitario. Con ello, posibilita relacionar teoría y práctica.

En la Tabla 2 recogemos las competencias pretendidas con la superación de esta asignatura. 
Actividades para fomentar el desarrollo socioemocional de Educación Infantil
CG5 Saber promover la adquisición de hábitos en torno a la autonomía, la libertad, la curiosidad, la observación, la experimentación, la imitación, la aceptación de normas y de límites, el juego simbólico y heurístico

CG22 Atender las necesidades de los estudiantes y transmitir seguridad, tranquilidad y afecto

CE011 Conocer la dimensión pedagógica de la interacción social en la escuela y saber promover la participación en actividades colectivas, el trabajo cooperativo y el esfuerzo individual

CE013 Diseñar contextos y actividades de aprendizaje para el adecuado desarrollo de las diferentes habilidades socioemocionales

CE017 Fomentar el desarrollo socioemocional de los estudiantes como vía para abordar la convivencia en los contextos escolares

- Inteligencia emocional y comunicación eficaz en el docente de Ed. Infantil (asignatura 3): la última de las asignaturas optativas de la mención se cursa en el primer semestre del $4^{\circ}$ curso, simultaneada con el Prácticum II. En este caso, el desarrollo de las prácticas del alumnado le exige la puesta en práctica de actividades de mención (es decir, el alumnado diseña y lleva a la práctica algunos de los contenidos trabajados).

El contenido de la asignatura se enfoca en este caso al propio alumno de magisterio. Procuramos fomentar el autoanálisis personal (autoestima, resiliencia, estilo comunicativo y relacional) de modo que se optimice el rendimiento como docentes y se disfrute de la profesión desde un punto de vista personal. La comunicación y el trabajo en equipo son dos de las áreas que abordamos.

En la Tabla 3 se recogen las competencias pretendidas con esta asignatura.

Tabla 3

Inteligencia emocional y comunicación eficaz en el docente de Ed. Infantil
CEO16 Establecer las relaciones entre el desarrollo de las habilidades socioemocionales y la calidad de las relaciones que se establecen con los iguales

CEO14 Disponer de recursos metodológicos y estrategias para favorecer la educación socioemocional en el aula

CEO15 Dominar las habilidades socioemocionales en el trato y relación con la familia de cada estudiante y con el conjunto de las familias

CEO18 Promover la mejora de la función docente a través del entrenamiento de las habilidades de comunicación y emocionales de los profesores

CEO20 Reflexionar sobre la importancia de abordar el desarrollo socioemocional en la educación infantil

CEO21 Valorar la importancia del trabajo en equipo y fomentar la colaboración con otros profesionales y agentes sociales 


\section{Conclusiones}

La mención Comportamiento prosocial y habilidades socioemocionales en Educación Infantil se imparte desde el curso 2012-2013 (primer curso académico que se matriculaba en tercero el alumnado desde el comienzo de los estudios de Grado).

Como ha podido apreciarse en el desarrollo del artículo, en dicha mención se aborda el tema de la Inteligencia Emocional desde un enfoque holístico, que comienza analizando conceptos e ideas de base relevantes en relación con el desarrollo socioemocional y, después, se sitúa en un marco más práctico: comenzamos con el niño en etapa escolar como objeto de intervención y continuamos con la figura del docente como objeto de autoanálisis. En la Figura 1 ofrecemos un diagrama con la visión de conjunto de las asignaturas que conforman la mención.

Desde el comienzo de su impartición, no ha dejado de haber alumnos interesados en matricularse, por lo que podemos suponer que es un tema que resulta atractivo, ya que hablamos de asignaturas optativas.

Por las características de la metodología que utilizamos (diarios de campo, portafolios, análisis de situaciones reales, dinámicas grupales...), el contacto que mantenemos con el alumnado es muy cercano, con diálogo constante y evaluaciones y autoevaluación frecuentes. Por ello, hemos podido constatar curso tras curso que la mención es muy valorada en cuanto a la utilidad personal que se le atribuye: el alumnado siente que se trata de una temática que se relaciona con su bienestar personal. Además, tienden a buscar aplicaciones en su contextos cotidianos (familiares, laborales...) por lo que se corrobora la impresión anterior.

Además, se comprueba mediante las calificaciones que obtienen los alumnos en dichas asignaturas, que el resultado alcanzado es altamente positivo y la implicación muy alta. A continuación, presentamos en la Tabla 4 dichas calificaciones.

\section{Figura 1}

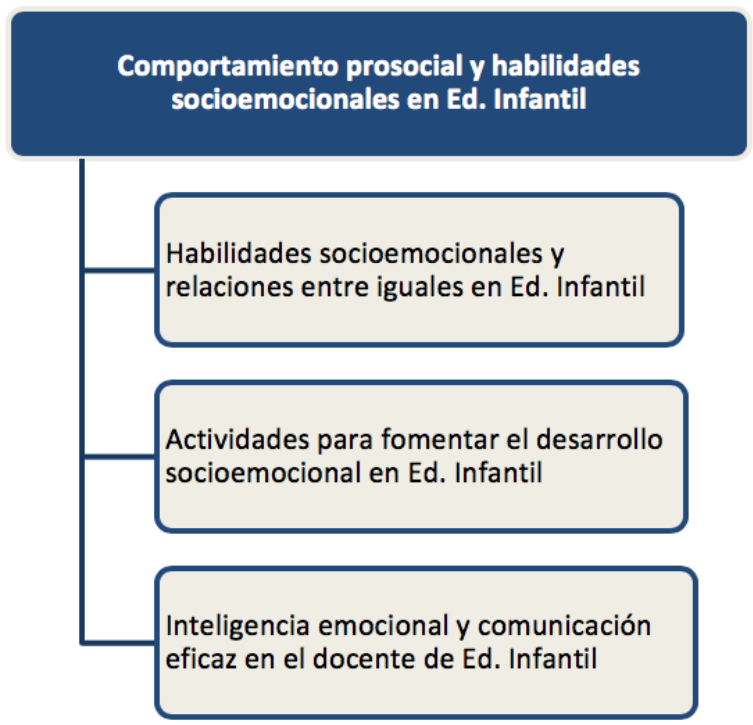




\begin{tabular}{|c|c|c|c|}
\hline & AsIGNATURA 1 & ASIGNATURA 2 & AsIGNATURA 3 \\
\hline \multirow[t]{4}{*}{$2016-17$} & Aprobada: $23 \%$ & Aprobada: $7.7 \%$ & Aprobada: $21.4 \%$ \\
\hline & Notable: $53 \%$ & Notable: $76.9 \%$ & Notable: $35.7 \%$ \\
\hline & Sobresaliente: $23 \%$ & Sobresaliente: $15.4 \%$ & Sobresaliente: $35.7 \%$ \\
\hline & Matrícula de Honor: 0 & Matrícula de Honor: 0 & Matrícula de Honor: $7.1 \%$ \\
\hline \multirow[t]{4}{*}{$2017-18$} & Aprobada: $37.5 \%$ & Aprobada: 50\% & Aprobada: $28.6 \%$ \\
\hline & Notable: $50 \%$ & Notable: $38.9 \%$ & Notable: $28.6 \%$ \\
\hline & Sobresaliente: $6.2 \%$ & Sobresaliente: $11.1 \%$ & Sobresaliente: $35.7 \%$ \\
\hline & Matrícula de Honor: 6.2\% & Matrícula de Honor: 0 & Matrícula de Honor: $7.1 \%$ \\
\hline
\end{tabular}

La valoración de las asignaturas (en las evaluaciones externas realizadas por la Universidad de Cádiz) es muy positiva, como recogemos en la Tabla 5.

\section{Tabla 5}

Resultados de valoración global de las asignaturas.

\begin{tabular}{cccc} 
& Asignatura 1 & Asignatura 2 & Asignatura 3 \\
\hline $2016-17$ & 4,8 & 4,8 & 4,9 \\
\hline $2017-18$ & 4,7 & 4,8 & 4,8 \\
\hline
\end{tabular}

No queremos dejar de señalar que somos conscientes de que el hecho de cursar una serie de asignaturas no proporciona necesariamente competencias socioemocionales pero sí que podemos sembrar el interés, incluso la duda, el autocuestionamiento de que algo podría hacerse de un modo diferente.

Por último, en el Centro de Magisterio Virgen de Europa también se imparte el Grado en Educación Primaria. Por su diseño y las características profesionalizantes de las menciones que ofrece, no permite abordar optativas en el campo de estudio de la Inteligencia Emocional. Desde las asignaturas que en este Grado se imparten desde nuestro Departamento de Psicología, se introducen algunos contenidos en relación con las inteligencias múltiples, aterrizando en la emocional (como es el caso de Psicología de la Educación) y se aborda el desarrollo socioemocional y su relación con el cerebro (en Psicología del Desarrollo); pero a todas luces, son contenidos mínimos, por lo que consideramos que hay una laguna importante en este área como además manifestaba algún sector del alumnado. Por ello, en los dos últimos cursos académicos, hemos ofrecido talleres en el marco de nuestro Programa de Orientación y Apoyo al Estudiante para los alumnos que quieran ampliar su formación de modo gratuito y voluntario. La oferta incluye tanto al alumnado de Educación Primaria como aquellos de Educación Infantil que deciden no cursar la mención. 
Consideramos que el estudio de este campo puede hacer grandes aportaciones al desarrollo personal y, en particular, a la profesión docente, por lo que estamos muy satisfechas de poder trabajar en él. Como señala Casas (2009), las competencias socioemocionales se desarrollan aprendiendo una serie de habilidades prácticas y específicas.

\section{REFERENCIAS BIBLIOGRÁFICAS}

Bayón, A. (2015). Competencia emocional docente ¡la revolución interior!. Padres y maestros, 361, 55-61.

Bermejo, L. (2016). Bienestar docente. Estrategias para una vida emocionalmente más saludable en el trabajo de profesor. Padres y maestros, 368, 18-23.

Casas, A. (2009). Las emociones en Educación Infantil. Manual para Ed. Infantil: orientaciones y recursos 0-6 años. Madrid: Wolters Kluwer España.

Gardner, H. (1999). Intelligence Reframed: Multiple Intelligences for the 21st Century. New York: Basic Books.

Goleman, D. (1998). Inteligencia Emocional. Barcelona: Kairós.

Informe de la UNESCO de la Comisión Internacional sobre la educación para el siglo XXI. Recuperado el 3/junio/2018 de www.unesco.org/education/pdf/DELORS_S.PDF

López Cassà, E. (2012). Educación emocional. Programa para 3-6 años. Madrid: Wolters-Kluwer.

Maganto, C. y Maganto, J. (2010). Cómo potenciar las emociones positivas y afrontar las negativas. Madrid: Pirámide.

Mayer, J. D. y Salovey, P. (1997). What is emotional intelligence? En P. Salovey y D. Sluyter (Eds.), Emotional Development and Emotional Intelligence: Implications for Educators (pp. 3-31). New York: Basic Books.

Navarro, J. I. y Martín, C. (coords) (2010). Psicología de la educación para docentes. Madrid: Pirámide.

Vaello, J. (2011). El profesor emocionalmente competente. Barcelona: Graó.

VVAA. (2010). La salud física y emocional del profesorado. Barcelona: Graó.

\section{INFORMACIÓN SOBRE LAS AUTORAS}

Claudia Ruiz López. Licenciada en Psicología por la Universidad de Granada y Doctora en Ciencias de la Educación por la Universidad de Cádiz. Desde 1995 es Profesora Titular Universitaria de diversas asignaturas de los Grados de Magisterio en Educación Infantil y Educación Primaria relacionadas con la Psicología de la Educación y del Desarrollo, los aspectos socioemocionales y las diferencias en el aprendizaje, en el Centro de Magisterio "Virgen de Europa" de La Línea de la Concepción (adscrito a la Universidad de Cádiz). Ha venido desempeñando diversos cargos y funciones académicas y de gestión en dicho centro. Actualmente es encargada del Área Departamental de Psicología y del Área de Información pública. Ha formado parte del grupo de investigación GIETE 
(código HUM-159) y sus intereses de investigación abarcan la Inteligencia emocional del docente así como la Psicología de la Educación y del Desarrollo.

$\triangle$ claudiaruizlopez@magisteriolalinea.com

Luisa Ma García Salas. Licenciada en Psicología por la Universidad de Málaga y Doctora en Psicología por la Universidad de Cádiz. Diplomada como Profesora de EGB (especialidad “Ciencias") por la Universidad de Cádiz. Desde octubre de 1990 hasta la fecha, Profesora Titular Universitaria del Área de Psicología Evolutiva y de la Educación en el Centro de Magisterio "Virgen de Europa" de La Línea de la Concepción (adscrito a la Universidad de Cádiz) de diversas asignaturas de la Diplomatura de Magisterio y de los actuales Grados en Educación Infantil y Educación Primaria. Ha sido miembro del Equipo Directivo del Centro y ha ocupado diversos cargos y funciones académicas. Actualmente es Coordinadora de Prácticas externas curriculares de los Grados en Ed. Infantil y Ed. Primaria. Ha sido ponente en diversos cursos y talleres relacionados con las áreas de Psicología y Educación. Sus intereses de investigación son la Inteligencia emocional en la formación inicial universitaria, así como las Dificultades de Aprendizaje e Intervención psicoeducativa. 